\title{
Comparison with Magnetic Resonance Three-Dimensional Sequence for Lumbar Nerve Root with Intervertebral Foramen
}

\author{
Hiroyuki Takashima ${ }^{1,2}$, Tsuneo Takebayashi ${ }^{2}$, Hiroki Shishido ${ }^{1}$, Mitsunori Yoshimoto ${ }^{2}$, \\ Rui Imamura ${ }^{1}$, Yoshihiro Akatsuka ${ }^{1}$, Yoshinori Terashima ${ }^{2}$, Hiroyoshi Fujiwara ${ }^{3}$, \\ Masateru Nagae ${ }^{3}$, Toshikazu Kubo ${ }^{3}$, Toshihiko Yamashita ${ }^{2}$ \\ ${ }^{1}$ Division of Radiology and Nuclear Medicine, Sapporo Medical University Hospital, Sapporo, Japan \\ ${ }^{2}$ Department of Orthopedic Surgery, Sapporo Medical University School of Medicine, Sapporo, Japan \\ ${ }^{3}$ Department of Orthopedic Surgery, Graduate School of Medical Science, Kyoto Prefectural University of Medicine, Kyoto, Japan
}

Study Design: Prospective study based on magnetic resonance (MR) imaging of the lumbar spinal root of the intervertebral foramen. Purpose: This study was to compare MR three-dimensional (3D) sequences for the evaluation of the lumbar spinal root of the intervertebral foramen.

Overview of Literature: The diagnosis of spinal disorders by MR imaging is commonly performed using two-dimensional T1- and T2-weighted images, whereas 3D MR images can be used for acquiring further detailed data using thin slices with multi-planar reconstruction.

Methods: On twenty healthy volunteers, we investigated the contrast-to-noise ratio (CNR) of the lumbar spinal root of the intervertebral foramen with a 3D balanced sequence. The sequences used were the fast imaging employing steady state acquisition and the coherent oscillatory state acquisition for the manipulation of image contrast (COSMIC). COSMIC can be used with or without fat suppression (FS). We compared these sequence to determine the optimized visualization sequence for the lumbar spinal root of the intervertebral foramen.

Results: For the CNR between the nerve root and the peripheral tissue, these were no significant differences between the sequences at the entry of foramen. There was a significant difference and the highest CNR was seen with COSMIC-FS for the intra- and extraforamen.

Conclusions: In this study, the findings suggest that the COSMIC-FS sequences should be used for the internal or external foramen for spinal root disorders.

Keywords: Lumbar nerve root; Intervertebral foramen; Magnetic resonance imaging; Three-dimensional; Coherent oscillatory state acquisition for the manipulation of image contrast; Fast imaging employing steady state acquisition

\section{Introduction}

Lumbar radiculopathy is classified by the site of its oc- currence, which includes the lateral recess, intervertebral foramen, and external intervertebral foramen zones. Previous studies [1-6] regarding diagnostic imaging tech-

\footnotetext{
Received Feb 11, 2015; Revised Mar 9, 2015; Accepted Mar 22, 2015

Corresponding author: Tsuneo Takebayashi

Department of Orthopedic Surgery, Sapporo Medical University School of Medicine,

South- 1, West-16, Chuo-ku, Sapporo 060-8543, Japan

Tel: +81-11-611-2111, Fax: +81-11-641-6026, E-mail: takebaya@sapmed.ac.jp
} 
Table 1. Scan parameters of FIESTA, COSMIC, and COSMIC-FS

\begin{tabular}{lccc} 
Variable & FIESTA & COSMIC & COSMIC-FS \\
Repetition time (msec) & 5.2 & 5.2 & 5.2 \\
\hline Echo time (msec) & 1.6 & 2.6 & 2.6 \\
\hline Band width (kHz) & 50.0 & - & - \\
Resolution (mm) & $0.625 \times 0.625 \times 1.0$ & $2025 \times 0.625 \times 1.0$ & 1.0 \\
Acquisition time (sec) & 216 & 203 & 207 \\
\hline
\end{tabular}

FIESTA, fast imaging employing steady state acquisition; COSMIC, coherent oscillatory state acquisition for the manipulation of image contrast; COSMIC-FS, COSMIC-fat suppression.

niques, including those for external intervertebral foramen lesions, which commonly prove as far-out-syndrome, have been reported [7]. However, because it can be difficult to diagnose these lesions by conventional magnetic resonance imaging (MRI), a more specific imaging technique is needed.

Common spinal MRI sequences include the twodimensional (2D) T1- and T2-weighted sagittal and axial planes $[3,6]$. Cerebrospinal fluid (CSF) exhibits high signals, whereas the intradural nerves exhibit low signals on T2-weighted images. Three-dimensional (3D) MRI can be used to acquire detailed morphologic data because its settings can be adjusted to obtain thin slices with a high signal-to-noise ratio (SNR), and multiplanar reconstruction (MPR) allows images to be created from the original plane in either the coronal, sagittal, or oblique plane. It therefore enables observations from multiple directions [2,8-10]. A technique called 3D fast-spin echo, which has been recently introduced, is effective for rendering nerve roots at high resolution, but the long acquisition time required for the imaging technique is a problem when patients are experiencing pain $[6,11]$.

Furthermore, the gradient-echo sequence, with multiple echo collection, improves the $\mathrm{T} 2$ contrast between nerve roots and intervertebral foramen stenosis $[12,13]$. The 3D balanced sequence renders the lumbar nerve roots with 3D collection performed over a short acquisition time [3,6,14-16]. Fast imaging employing steady state acquisition (FIESTA) is a 3D sequence with high fluid sensitivity that enables the high resolution of small intracranial structures and joints. It provides exquisite contrast that is ideally equated for visualization of the internal auditory canal. It is also ideally suited for T2 imaging through the cervical spine region. In contrast, coherent oscillatory state acquisition for the manipulation of image contrast
(COSMIC) uses a unique "pre" steady-state imaging technique to deliver optimized visualization of soft-tissue structures adjacent to bony structures. However, there are no reports describing which sequences are better-suited to rendering nerve roots in the intervertebral foramina. Our purpose in this study was to use FIESTA and COSMIC to investigate the rendering of intervertebral foramen nerve roots.

\section{Materials and Methods}

All subjects provided written informed consent to participate in this study, which was conducted with the approval of the ethics committee. We evaluated 20 healthy volunteers (13 males, 7 females; mean age, $44 \pm 7.3$ years), who had no history of leg pain or lumbar surgery.

The equipment used was a Signa HDxt 1.5T (GE Healthcare, Milwaukee, WI, USA) with a spine coil. The 3D balanced sequences acquired were FIESTA and COSMIC. COSMIC can be used with or without fat suppression (FS). The parameters used in the present study are shown in Table 1.

Ziostation 2 (Ziosoft Inc., Tokyo, Japan) was used to create a MPR that followed the right L5 nerve root (Fig. 1). The nerve root and surrounding tissue signal intensities were measured by areas perpendicular to the L5 nerve root in the intervertebral foramen entrance, intervertebral foramen, and intervertebral foramen external zones (Fig. 2). The tissue or fluid adjacent to the nerve root was CSF in the intervertebral foramen entrance zone, the pedicle in the intervertebral foramen zone, and the fat in the intervertebral foramen external zone. The contrast-to-noise ratio (CNR) of these tissues with the nerve root was calculated and the results were compared by use of FIESTA, COSMIC, and COSMIC-FS sequences. The CNR was 

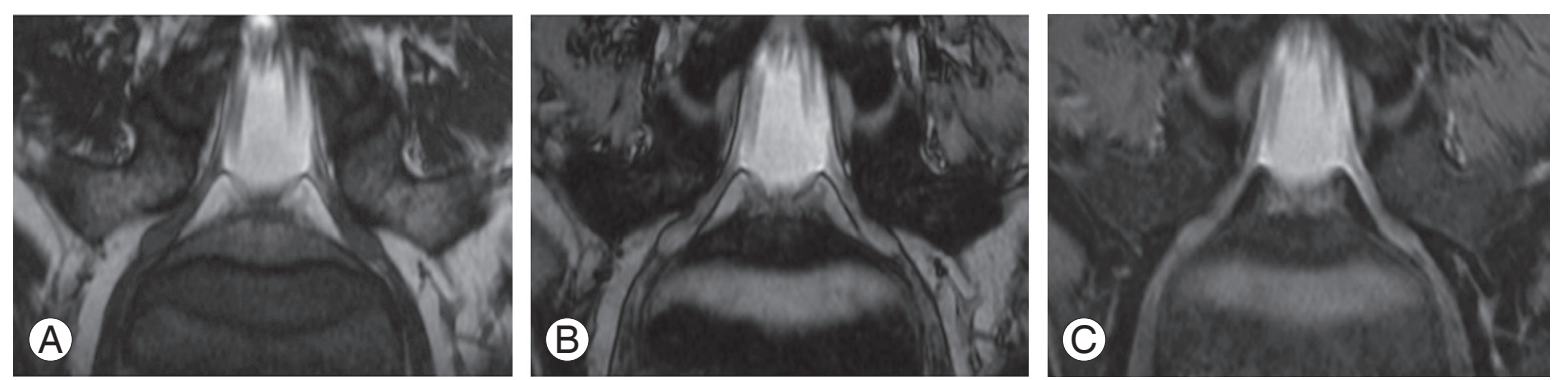

Fig. 1. Multiplanar reconstruction of $L 5$ nerve root with fast imaging employing steady state acquisition (FIESTA), coherent oscillatory state acquisition for the manipulation of image contrast (COSMIC), and COSMIC-fat suppression (FS). (A) FIESTA, (B) COSMIC, (C) COSMIC-FS.

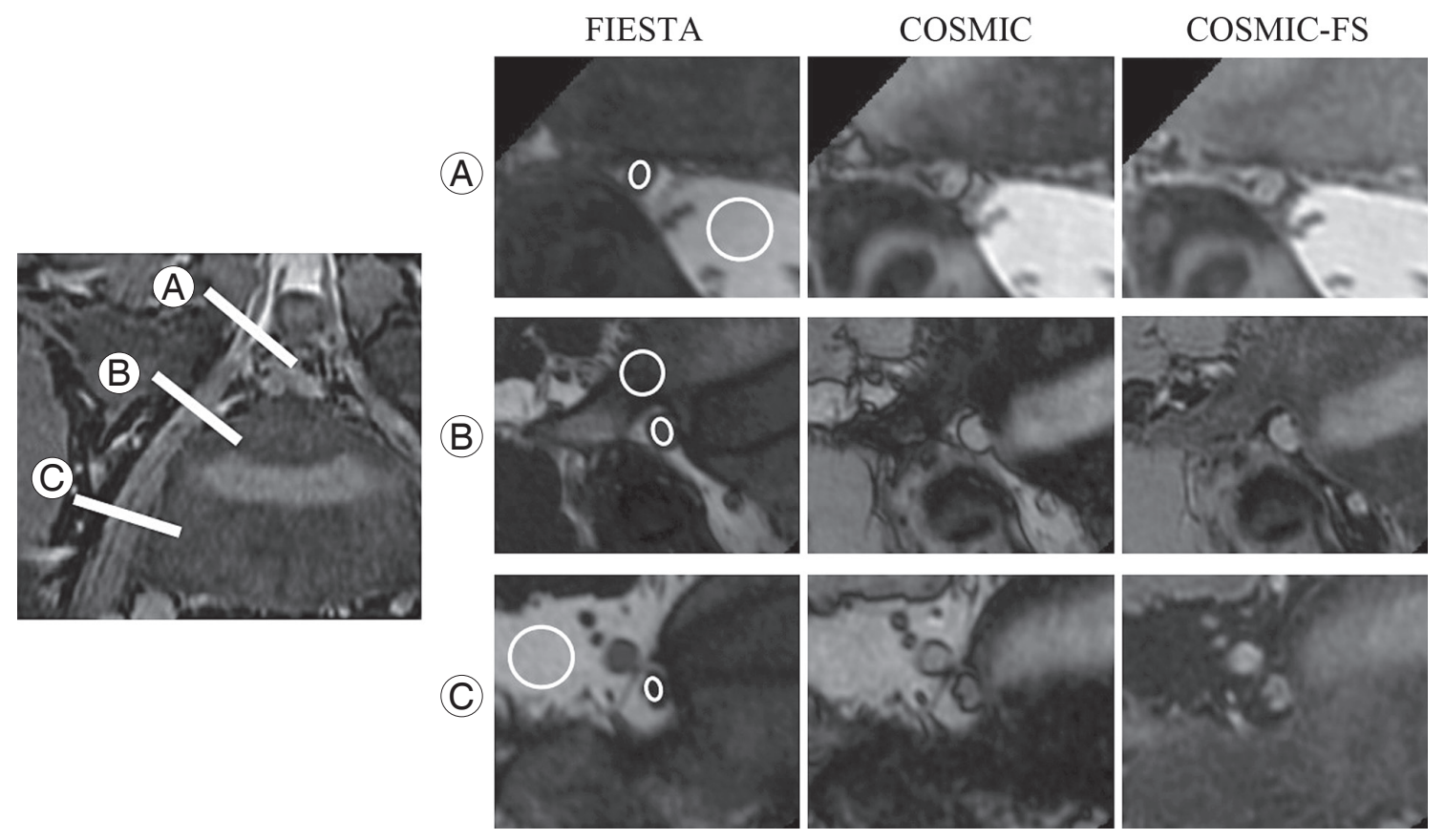

Fig. 2. Position of the region of interests (ROIs) and image of the investigation slice with fast imaging employing steady state acquisition (FIESTA), coherent oscillatory state acquisition for the manipulation of image contrast (COSMIC), and COSMIC-fat suppression (FS). The mean value of the ROls were measured in the root tissue, peripheral tissue, and standard deviation of peripheral tissue, respectively. The images of investigated slices for the entry of the foramen, intra-foramen, and extra-foramen with L5 nerve root are shown. ROls of nerve root and cerebrospinal fluid for entry of foramen (A). ROls of nerve root and pedicle for intraforamen (B). ROls of nerve root and sacral bone for extra-foramen (C).

calculated from the following formula:

$$
\mathrm{CNR}=\frac{\mid \mathrm{SI}(\text { root })-\mathrm{SI}(\text { peripheral }) \mid}{\mathrm{SD}(\text { peripheral })}
$$

SI, signal intensity; SD, standard diviation.

The data were analyzed statistically by use of MedCalc ver. 10.2 (MedCalc software, Ostend, Belgium). The Wilcoxon signed rank test was used for significance testing. A $p$-value of $<0.05$ was considered to indicate statistical significance.

\section{Results}

No significant differences were observed in the CNR between the nerve root and CSF in the intervertebral foramen entrance zone as calculated by all of the sequences (Fig. 3). The CNR between the nerve root and pedicle of the vertebral arch in the intervertebral foramen (Fig. 4) and that between the nerve root and the fat in the intervertebral foramen zone tended to be the highest when measured by COSMIC-FS (Fig. 5). 


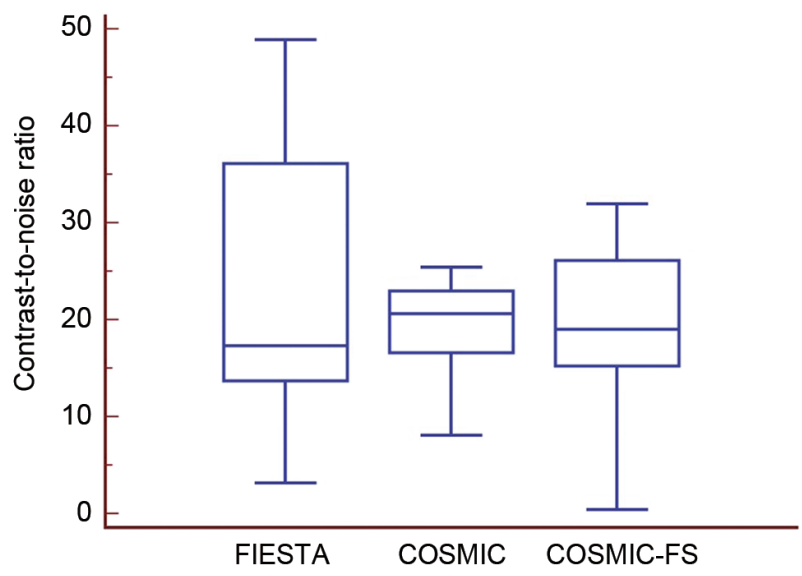

Fig. 3. Contrast-to-noise ratio (CNR) of nerve root and cerebrospinal fluid (CSF) with fast imaging employing steady state acquisition (FIESTA), coherent oscillatory state acquisition for the manipulation of image contrast (COSMIC), and COSMIC-fat suppression (FS) for the entry of foramen. The CNR between the nerve root and CSF with entry of foramen showed no significant difference between sequences.

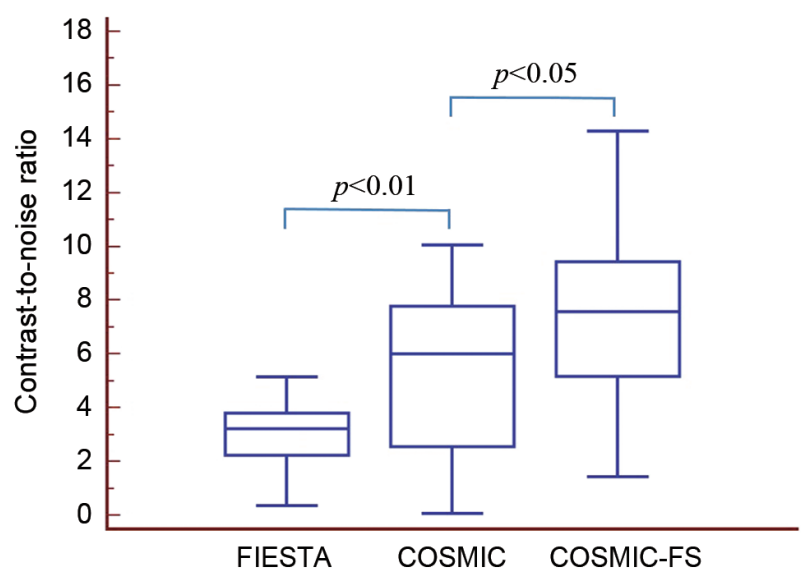

Fig. 4. Contrast-to-noise ratio (CNR) of nerve root and pedicle with fast imaging employing steady state acquisition (FIESTA), coherent oscillatory state acquisition for the manipulation of image contrast (COS$\mathrm{MIC}$ ), and COSMIC-fat suppression (FS) for the intra-foramen. The CNR between the nerve root and pedicle at the intra-foramen were significantly different between sequences; the highest was COSMICFS $(p<0.05)$

\section{Discussion}

The diagnosis of nerve root compression and spinal canal stenosis by normal 2D lumbar vertebra MRI can be difficult. Furthermore, it is often challenging to identify the level responsible for certain conditions, such as epidural radiculopathy $[3,6,17]$.

In the present study, we compared extradural renderings of the nerve root by the FIESTA and COSMIC sequences. 3D sequences with high SNRs are excellent for

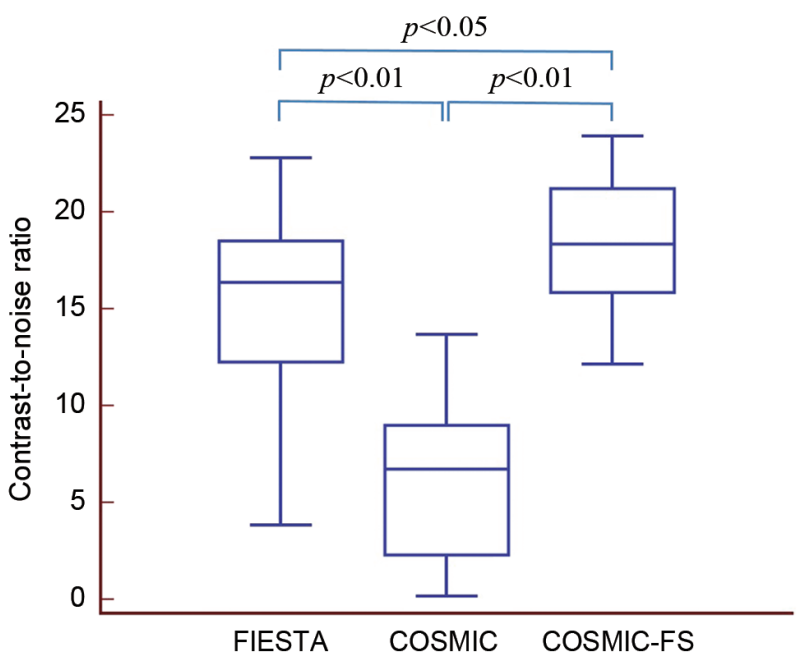

Fig. 5. Contrast-to-noise ratio (CNR) of nerve root and fat with fast imaging employing steady state acquisition (FIESTA), coherent oscillatory state acquisition for the manipulation of image contrast (COSMIC), and COSMIC-fat suppression (FS) for the extra-foramen. The CNR between the nerve root and fat at the extra-foramen were significantly different; the highest was COSMIC-FS $(p<0.05)$.

detecting morphologic changes, and the high spatial resolution in these sequences makes MPR possible without degrading the image [18-20]. SNR is equal to the ratio of the average signal intensity over the standard deviation of the noise and an important reference of MRI. Because of the contrast in the FIESTA, water components and blood appear as high signals, but the soft-tissue signals with short $\mathrm{T}_{2}$ values give a lower signal intensity $[21,22]$. The CNR between the nerve root and CSF in the intervertebral foramen entrance zone showed no significant difference among these sequences and proved to be the same with each of them regarding the assessment of the intradural zone where CSF is present.

CNR is the relationship of signal intensity differences between two regions, scaled to image noise, and a summary of SNR and contrast. The FIESTA provided little contrast between the peripheral tissues and the nerve root and its structures, such as bone or intervertebral discs, and it was difficult to identify the nerve root. The COSMIC is performed before echo sampling reaches a steady state and exhibits greater soft-tissue contrast [2]. This improves the CNR and SNR of cervical-spine tissue including the spinal cord, vertebral discs, and nerve root canal, as well as the contrast between CSF and nerve roots [23]. In this study, in the comparison of COSMIC and COSMIC-FS on the intra- and extra-foramen, the CNR between the nerve root and peripheral tissue was signifi- 
cantly higher for COSMIC-FS than for COSMIC. Accordingly, COSMIC-FS appears to provide improved contrast between the nerve root and intervertebral disc and bone for distinguishing the nerve root from the intervertebral foramina and the outside of the intervertebral foramina. COSMIC-FS also appeared to exhibit improved rendering in the intervertebral foramen zone and intervertebral external zone, where epidural fat is present, because the concomitant use of FS made the inhibition of chemical shift artifacts possible. The results of our study suggest that either sequence is useful for detecting intradural lesions, and that COSMIC-FS is superior for detecting nerve root lesions that are adjacent to soft-tissue inside and outside the intervertebral foramina, where there is little water.

However, a number of limitations should be mentioned. First, because we targeted healthy volunteers with no lesions in the lumbar vertebrae, we did not examine the rendering of actual lesions such as intervertebral disc hernias. Second, we were not able to compare the images obtained with FIESTA and COSMIC under the same conditions because it was impossible to use FS with FIESTA in this study. Third, other 3D sequences could not be investigated because we were only able to investigate the sequences possible with the equipment available.

\section{Conclusions}

We investigated lumbar nerve root visualization by using two types of 3D sequences. These sequences gave favorable results in the intervertebral foramen entrance zone, and the COSMIC-FS gave favorable results in the intervertebral foramen and intervertebral external zones. In this study, it was suggested that the $3 \mathrm{D}$ COSMIC was a suitable sequence to visualize the intervertebral foramen for spinal root disorders.

\section{Conflict of Interest}

No potential conflict of interest relevant to this article was reported.

\section{References}

1. Lou ZH, Qu JR, Li HL, et al. Optimal technique of three-dimensional MRI of the lumbar nerve root and its radicular vein in normal and lumbar disc herniation patients. Chin Med J (Engl) 2011;124:1802-6.
2. Tanitame K, Tanitame N, Tani C, et al. Evaluation of lumber nerve root compression using thin-slice thickness coronal magnetic resonance imaging: three-dimensional fat-suppressed multi-shot balanced non-steady-state free precession versus threedimensional T1-weighted spoiled gradient-recalled echo. Jpn J Radiol 2011;29:623-9.

3. Bieri O, Scheffler K. Fundamentals of balanced steady state free precession MRI. J Magn Reson Imaging 2013;38:2-11.

4. Byun WM, Ahn SH, Ahn MW. Value of 3D MR lumbosacral radiculography in the diagnosis of symptomatic chemical radiculitis. AJNR Am J Neuroradiol 2012;33:529-34.

5. Byun WM, Jang HW, Kim SW. Three-dimensional magnetic resonance rendering imaging of lumbosacral radiculography in the diagnosis of symptomatic extraforaminal disc herniation with or without foraminal extension. Spine (Phila Pa 1976) 2012;37: 840-4.

6. Danagoulian GS, Qin L, Nayak KS, et al. Comparison of wideband steady-state free precession and T(2)weighted fast spin echo in spine disorder assessment at 1.5 and 3 T. Magn Reson Med 2012;68:1527-35.

7. Wiltse LL, Guyer RD, Spencer CW, Glenn WV, Porter IS. Alar transverse process impingement of the L5 spinal nerve: the far-out syndrome. Spine (Phila $\mathrm{Pa}$ 1976) 1984;9:31-41.

8. Meindl T, Wirth S, Weckbach S, Dietrich O, Reiser M, Schoenberg SO. Magnetic resonance imaging of the cervical spine: comparison of 2D T2-weighted turbo spin echo, 2D T2* weighted gradient-recalled echo and 3D T2-weighted variable flip-angle turbo spin echo sequences. Eur Radiol 2009;19:713-21.

9. Ross JS. MR imaging of the cervical spine: techniques for two- and three-dimensional imaging. AJR Am J Roentgenol 1992;159:779-86.

10. Wutke R, Fellner FA, Fellner C, Stangl R, Dobritz M, Bautz WA. Direct MR arthrography of the shoulder: $2 \mathrm{D}$ vs. $3 \mathrm{D}$ gradient-echo imaging. Magn Reson Imaging 2001;19:1183-91.

11. Vargas MI, Viallon M, Nguyen D, Beaulieu JY, Delavelle J, Becker M. New approaches in imaging of the brachial plexus. Eur J Radiol 2010;74:403-10.

12. Dorenbeck U, Schreyer AG, Schlaier J, Held P, Feuerbach S, Seitz J. Degenerative diseases of the cervical spine: comparison of a multiecho data image 
combination sequence with a magnetisation transfer saturation pulse and cervical myelography and CT. Neuroradiology 2004;46:306-9.

13. Vertinsky AT, Krasnokutsky MV, Augustin M, Bammer R. Cutting-edge imaging of the spine. Neuroimaging Clin N Am 2007;17:117-36.

14. Baskaran V, Pereles FS, Russell EJ, et al. Myelographic MR imaging of the cervical spine with a $3 \mathrm{D}$ true fast imaging with steady-state precession technique: initial experience. Radiology 2003;227:585-92.

15. VanDyke CW, Modic MT, Beale SM, Amartur S, Ross JS. 3D MR myelography. J Comput Assist Tomogr 1992;16:497-500.

16. Gjesdal KI, Storaas T, Geitung JT. A noncontrast-enhanced pulse sequence optimized to visualize human peripheral vessels. Eur Radiol 2009;19:110-20.

17. Jarvik JG, Deyo RA. Diagnostic evaluation of low back pain with emphasis on imaging. Ann Intern Med 2002;137:586-97.

18. Halldin K, Lind B, Ronnberg K, et al. Three-dimensional radiological classification of lumbar disc herniation in relation to surgical outcome. Int Orthop
2009;33:725-30.

19. Shen J, Wang HY, Chen JY, Liang BL. Morphologic analysis of normal human lumbar dorsal root ganglion by 3D MR imaging. AJNR Am J Neuroradiol 2006;27:2098-103.

20. Zhang Z, Song L, Meng Q, et al. Morphological analysis in patients with sciatica: a magnetic resonance imaging study using three-dimensional high-resolution diffusion-weighted magnetic resonance neurography techniques. Spine (Phila Pa 1976) 2009;34:E245-50.

21. Haacke EM, Wielopolski PA, Tkach JA, Modic MT. Steady-state free precession imaging in the presence of motion: application for improved visualization of the cerebrospinal fluid. Radiology 1990;175:545-52.

22. Markl M, Leupold J. Gradient echo imaging. J Magn Reson Imaging 2012;35:1274-89.

23. Shishido H, Takashima H, Takebayashi T, et al. Visualization of the foramen intervertebral nerve root of cervical spine with 3.0 tesla magnetic resonance imaging: a comparison of three-dimensional acquisition techniques. Nihon Hoshasen Gijutsu Gakkai Zasshi 2014;70:670-5. 